\title{
Flying Gardens: A Robotic Architectural Proposal for a Sessional Garden
}

\author{
KATELYN HANNIGAN \\ Kent State University
}

\section{JESSICA SCHULTZ}

Kent State University

\author{
EBRAHIM POUSTINCHI \\ Kent State University
}

Flying Gardens project is an architectural question about the concept of time and change/reconfiguration through revisiting the inside/outside problem in architecture. The project focuses on the inherently architectural qualities manifested through the relationship between static and animated architectural components as well as interiors exterior. Following a flat ontological approach to design the architecture, flying gardens project, studies the potentials of object-oriented design strategies, employing time and motion as vehicles for the conversation. Using an industrial robotic arm, as an animator, to illustrate the possibility of physical architectural animation, allows for formal and spatial but scale-less relationships to emerge.

Through multiple configurations, flying gardens revisits the concept of time and spatial organizations through day/night, different temperature, weather, and seasonal scenarios. This notion of time influences the inside/outside relationship and is been illustrated through the difference of the formal language. Use of flowers in the physical model, not only a represents an architectural quality of a garden-garden surface but also suggests textural/formal dialogues between inside/ outside and animated/static surfaces. The floral surfaces are projections of possible formal and architectural opportunities, such as apertures, and penalizations, mass/ground relationship to name a few.

Responding to Tom Wiscombe's calls for flat-ontology in architecture, ${ }^{1}$ this project seeks a non-hierarchical relationship between parts, where every part of the "architecture" works as an object. Inside of the object-independent from the design of the envelope, reflects the objecthood of the interiors. Ground-object works as a low-relief two and a half dimensional object that precisely interacts with the building object. Following this methodology, the flying gardens project closely revisits the concept of "super component." According to Tom Wiscombe, super-component an object that at the same time can act as an independent object and be part of another object. Through motion and reconfigurations, flying garden revisits this idea in a more literal way. In scenario one, the garden surface as an animated super component works as part of the building object to create a complete whole.
However, in the second scenario, the same component-garden surface, becomes an independent object that operates as an entrance garden. Reintroduction of the super component into the second position allows the once contained flowers with the spherical voids and varying degrees of thickness from inside the object to spew out and create different configurations to start the spatial conversation.

This literal motion-according to Greg Lynn's terminology, ${ }^{2}$ introduces interior surfaces as exteriors and vice-versa to maximize the spatial potentials through challenging form, configuration, part to part and part to whole relationships, surface qualities, and organizational strategies to name a few.

\section{ENDNOTES}

1 T. Wiscombe, "Discreteness, or Towards a Flat Ontology of Architecture," Project 3 (2014): 34-43.

2 G. Lynn, Animate FORM (New York: Princeton Architectural Press, 1999). 


\section{Flying Gardens}

A robotic architectural proposal for a sessional garden

[Hidden for Peer Review], [Hidden for Peer Review], [Hidden for Peer Review]
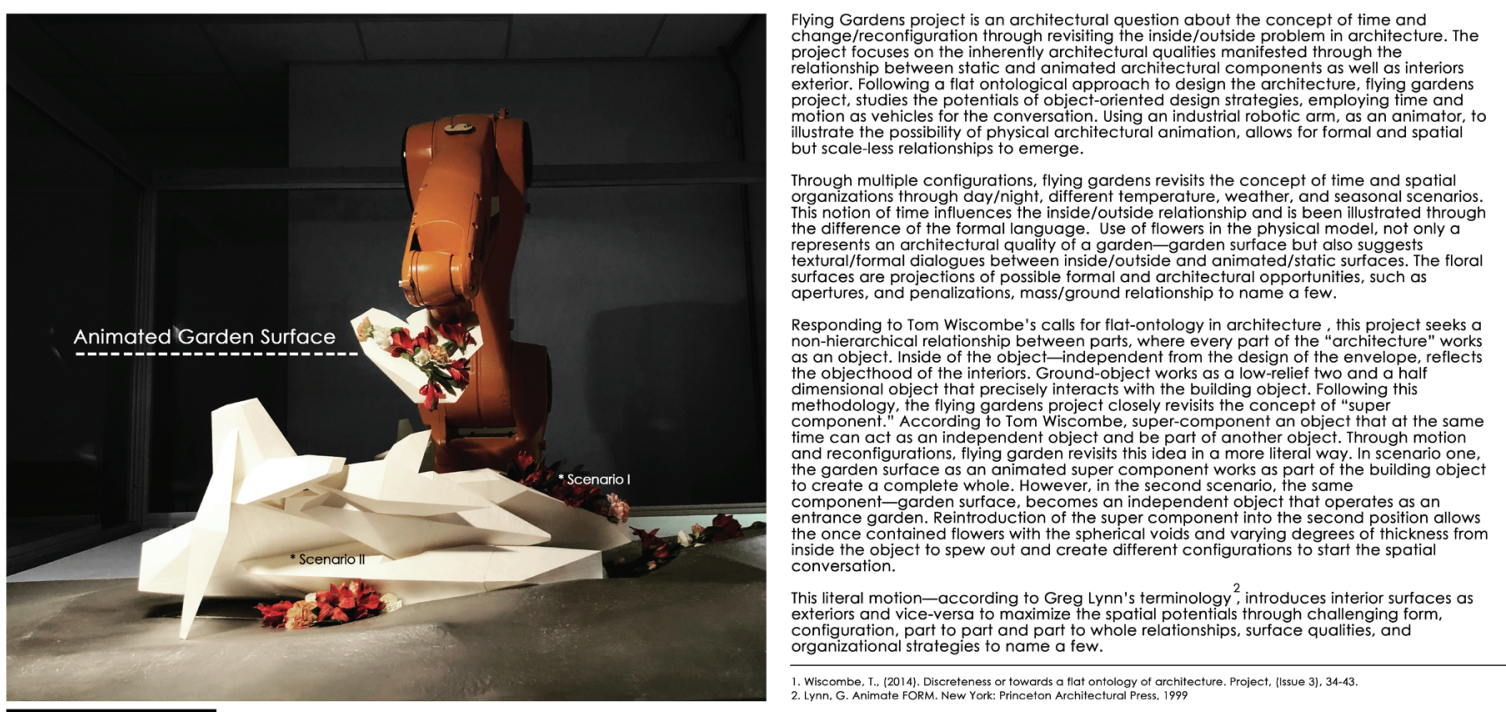

Making Process
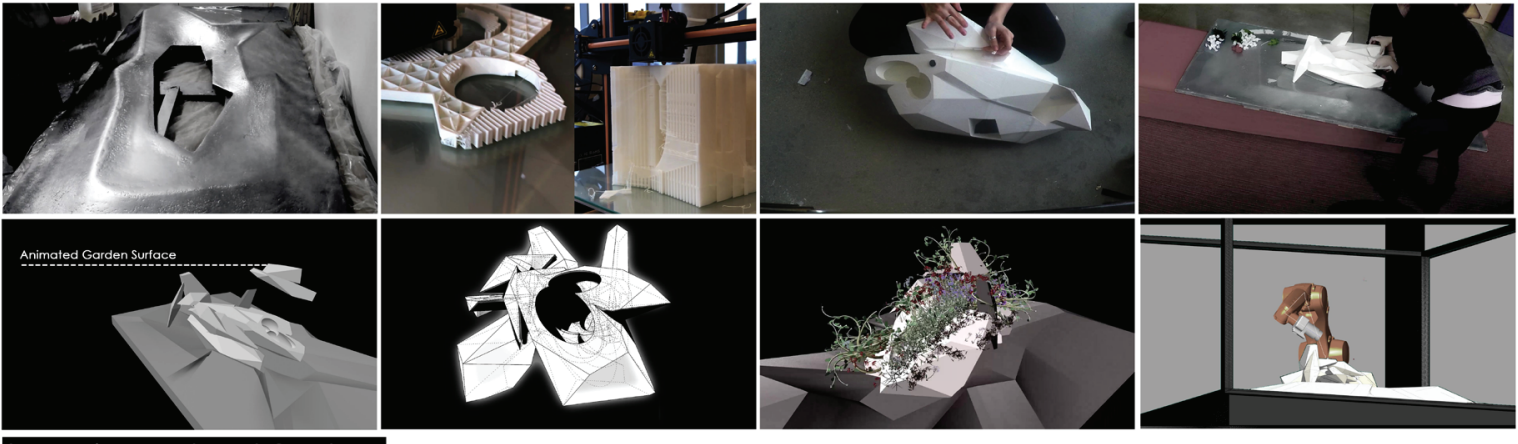

\section{Scenario I. Enclosed Garden}
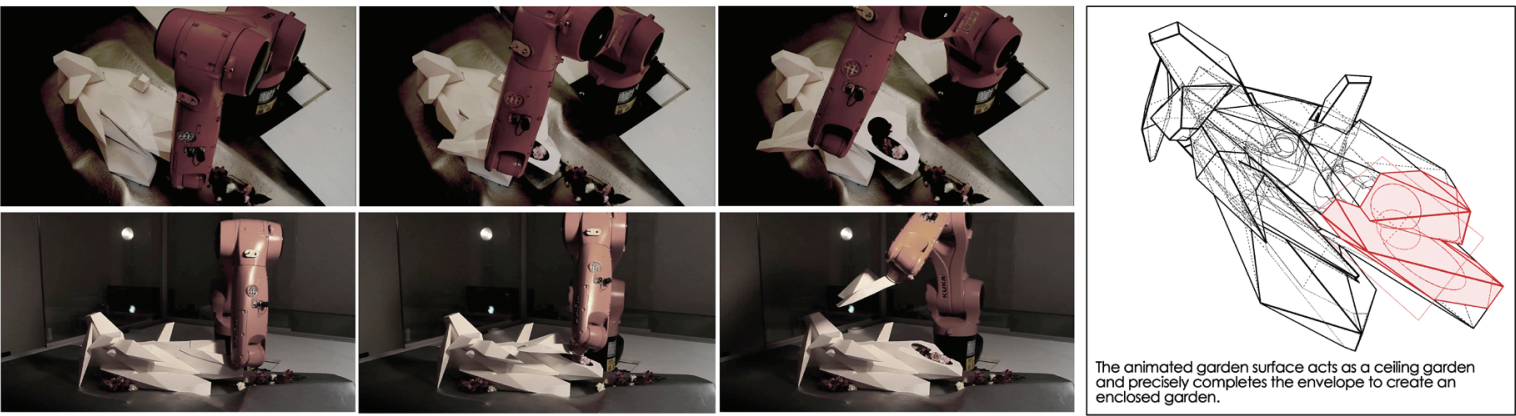

\section{Scenario II. Opne Garden/Entrance}
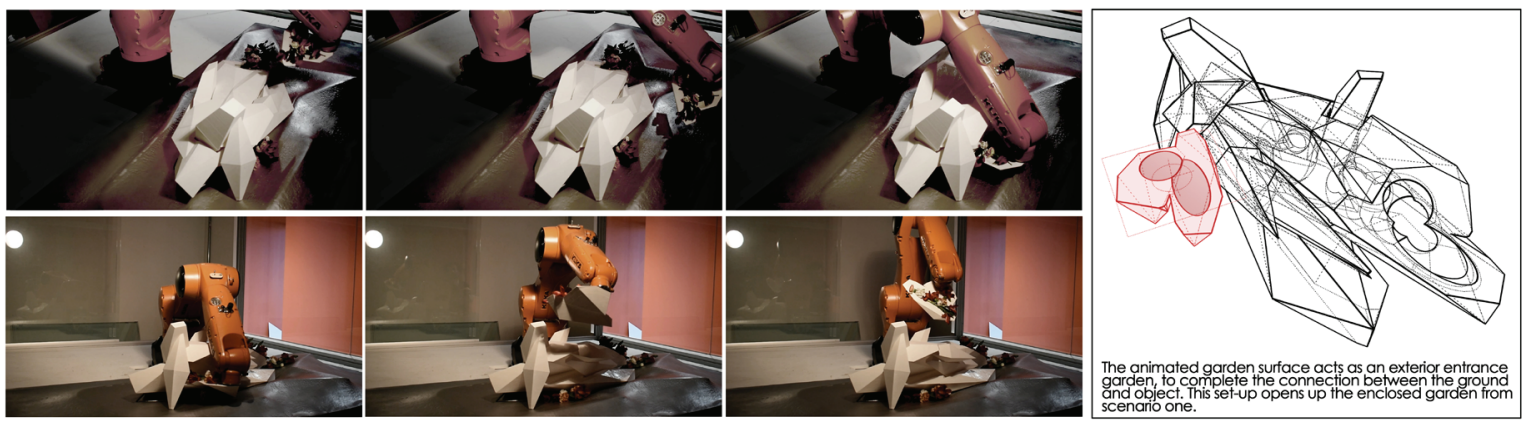